\title{
Endoscopic transcecal appendectomy: a new endotherapy for appendiceal orifice lesions $\square$
}

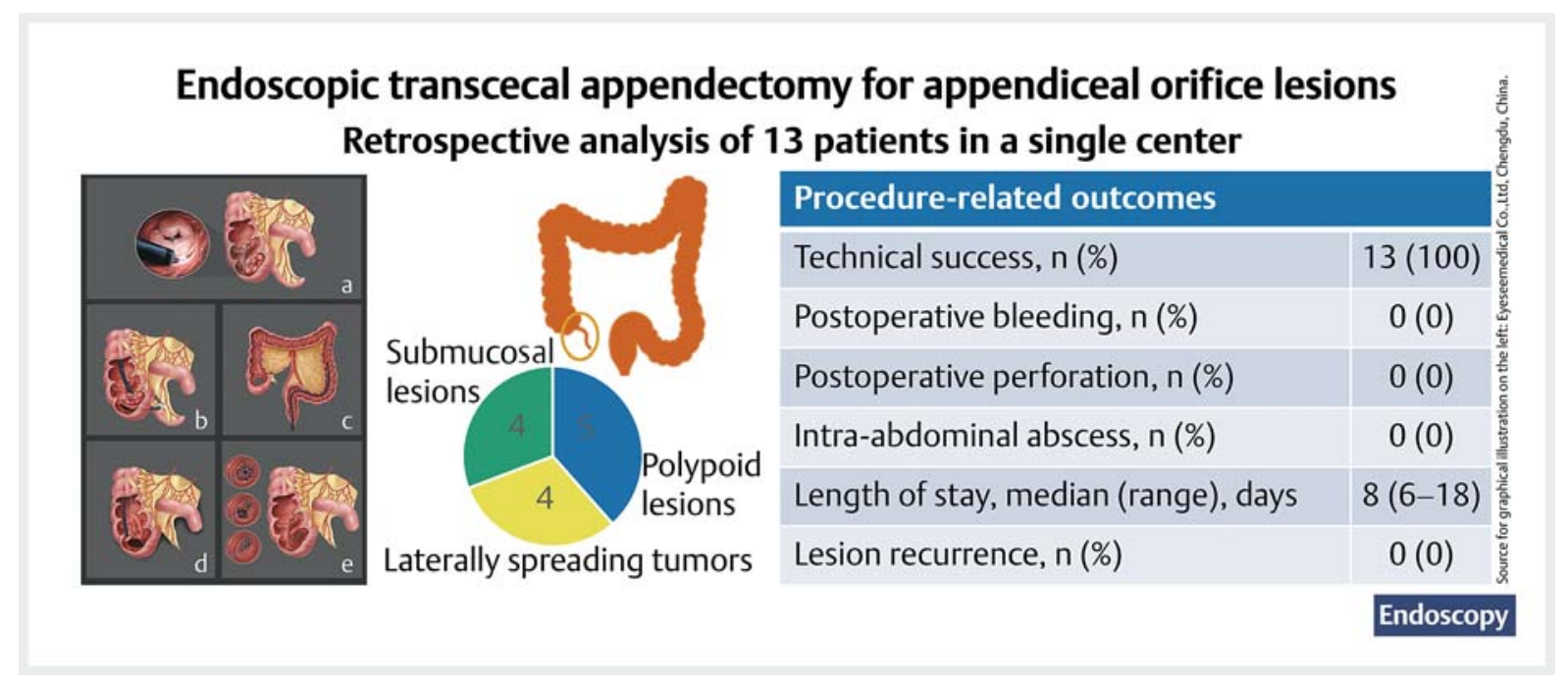

\section{다 (요우}

\section{Authors}

Linjie Guo ${ }^{1,{ }^{*}}$, Liansong Ye ${ }^{1,{ }^{*}}$, Yilong Feng ${ }^{1}$, Johannes Bethge ${ }^{2}$, Juliana Yang ${ }^{3}$, Stefan Schreiber ${ }^{2}$, Bing Hu ${ }^{1}$ ()

\section{Institutions}

1 Department of Gastroenterology, West China Hospital, Sichuan University, China

2 Department of Gastroenterology, University Medical Center Schleswig Holstein, Campus Kiel, Germany

3 The Vanderbilt University Medical Center, Nashville, Tennessee, United States

submitted 12.5.2021

accepted after revision 20.9.2021

published online 14.12.2021

\section{Bibliography}

Endoscopy 2022; 54: 585-590

DOI 10.1055/a-1675-2625

ISSN 0013-726X

(c) 2021. The Author(s). This is an open access article published by Thieme under the terms of the Creative Commons Attribution-NonDerivative-NonCommercial License, permitting copying and reproduction so long as the original work is given appropriate credit. Contents may not be used for commercial purposes, or adapted, remixed,

transformed or built upon. (https://creativecommons.org/ licenses/by-nc-nd/4.0/)

Georg Thieme Verlag KG, Rüdigerstraße 14,

70469 Stuttgart, Germany

$\bigoplus$ Fig. 1s

Supplementary material is available under https://doi.org/10.1055/a-1675-2625

\# Scan this QR-Code for the author commentary.

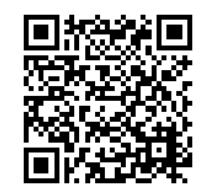

Corresponding author Bing Hu, MD, Department of Gastroenterology, West China Hospital, No. 37, Guoxue Alley, Wuhou, Chengdu, Sichuan 610041, China

hubingnj@163.com

\footnotetext{
* These authors contributed equally to this work.
} 


\section{ABSTRACT}

Background Endoscopic transcecal appendectomy (ETA) has been reported as a minimally invasive alternative procedure for lesions involving the appendiceal orifice. The aim of this case series study was to evaluate the feasibility, safety, and effectiveness of ETA for lesions at the appendiceal orifice.

Methods This retrospective study included consecutive patients with appendiceal orifice lesions who underwent ETA between December 2018 and March 2021. The primary outcome was technical success. The secondary outcomes included postoperative adverse events, postoperative hospital stay, and recurrence.
Results 13 patients with appendiceal orifice lesions underwent ETA during the study period. The median lesion size was $20 \mathrm{~mm}$ (range 8-50). Lesions morphologies were polypoid lesions $(n=5)$, laterally spreading tumors $(n=4)$, and submucosal lesions $(n=4)$. Technical success with complete resection was achieved in all 13 cases. There were no postoperative bleeding, perforation, or intra-abdominal abscess. The median length of hospital stay after ETA was 8 days (range 6-18). There was no tumor recurrence during a median follow-up of 17 months (range 1-28).

Conclusions ETA is feasible, safe, and effective for complete resection of appendiceal orifice lesions. Larger, multicenter, prospective studies are needed to further assess this technique.

\section{Introduction}

The advancement in endoscopic instruments has allowed endoscopic treatment to become the primary choice for treatment of various colorectal lesions such as polyps, laterally spreading tumors (LSTs), and submucosal lesions (SMLs) [1]. Endoscopic mucosal resection (EMR) and endoscopic submucosal dissection (ESD) have become the most commonly used methods for these colorectal lesions [2-4]. However, for lesions involving the appendiceal orifice, especially for lesions that invade deeply into the appendiceal lumen, complete resection with EMR or ESD is technically challenging because the distal margin cannot be fully visualized [2-4]. These types of lesions often require surgical interventions [5].

In recent years, endoscopic full-thickness resection (EFTR) using a specially designed EFTR device (FTRD; Ovesco Endoscopy, Tübingen, Germany) has been introduced to dissect lesions involving the appendiceal orifice [6-8]. Although it is a single-step and non-exposure procedure, the EFTR still cannot ensure complete resection of lesions with deep invasion into the appendiceal lumen, and may lead to a higher incidence of postoperative appendicitis [6-8]. Surgery may be needed for some of these patients owing to postoperative perforation or intrabdominal abscess [6-8]. In 2018, Liu et al. reported the first description of full-thickness resection of lesions involving the appendiceal orifice and simultaneous dissection of the appendix [9], known as the endoscopic transcecal appendectomy (ETA) technique. The ETA technique allows the appendiceal lesion to be completely resected regardless of its extension into the appendiceal orifice, and prevents the possibility for development of postoperative appendicitis. To date, the ETA technique has been applied to various appendiceal orifice lesions including colonic sessile serrated lesions, appendiceal retention cysts, appendiceal polyps, and chronic appendicitis [9-12]. However, previous studies on ETA have been generally small (four cases at most) [9-12].

The present retrospective case series study was designed to evaluate the feasibility, safety, and effectiveness of ETA in the management of lesions at the appendiceal orifice, including polypoid lesions, LSTs, and SMLs.

\section{Methods}

\section{Study design}

This retrospective case series study was conducted in a tertiary hospital (West China Hospital, Sichuan University, Chengdu, China). The study protocol was reviewed and approved by the Biomedical Research Ethics Committee of West China Hospital, Sichuan University.

\section{Patients}

Consecutive patients with appendiceal orifice lesions who underwent ETA in our hospital between December 2018 and March 2021 were retrospectively reviewed from our database of prospectively collected data. Patients with advanced carcinoma or with previous appendectomy were excluded. All patients received a preoperative consultation with detailed explanation of the pros and cons of different approaches including surgery, ESD, EFTR, and the novel ETA procedure. Informed consent to undergo the ETA procedure was obtained from all included patients.

\section{Procedures}

All ETA procedures were performed by an advanced endoscopist (B.H.), who had performed $\geq 400$ colorectal ESD procedures prior to the current study. All patients underwent strict bowel preparation to reduce intestinal contents and were treated under general anesthesia with intubation. Before the procedures, the enteric cavities were cleaned using sterilized water. All ETA procedures were performed using Olympus endoscopes (PCF-Q260JL/I; Olympus, Tokyo, Japan) and routine ESD instruments: insulated-tip knife (IT knife), dual knife, hook knife, a straight 4-mm clear cap, metal clips, and endoloop. A 20-mL syringe with $18-G$ needle was also available for abdominal decompression when the endoscope was introduced into the abdomen. 


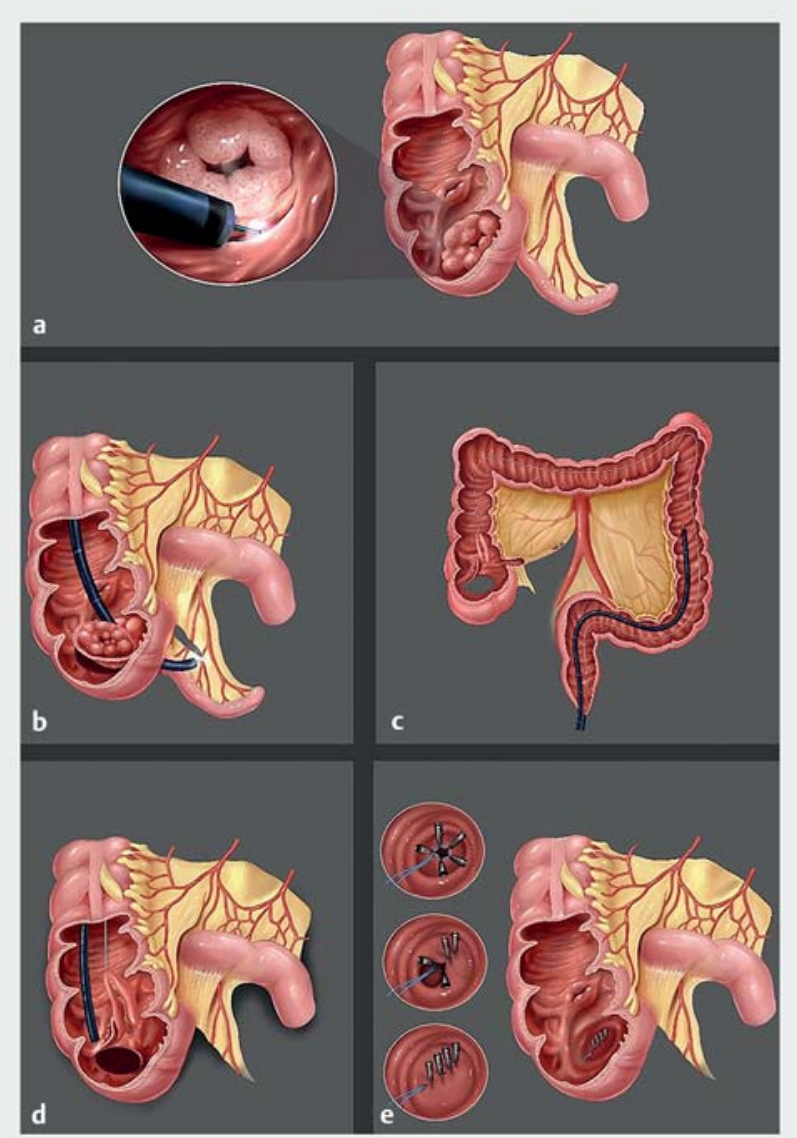

Fig. 1 Illustration of endoscopic transcecal appendectomy. a Near-circumferential full-thickness resection around the lesion after marking. $\mathbf{b}$ Introduction of the endoscope into the peritoneal cavity through the incision in order to dissect and cut off the mesoappendix and appendicular artery. c, $\mathbf{d}$ Snare-assisted traction of the partially dissected appendix for adequate exposure of the cutting line; a second endoscope was inserted for continuous dissection. e Closure of the defect using double endoscopic suture technique. Source: Eyeseemedical Co.,Ltd, Chengdu, China.

The ETA procedure involved the following steps ( $\bullet$ Fig.1, - Fig. 2, - Video 1): 1) circumferential marking of the lesion border using a dual knife with soft coagulation ERBE setting; 2) near-circumferential full-thickness resection around the lesion using dual knife and IT knife with Endocut setting; 3) introduction of the endoscope into the peritoneal cavity through the incision in order to dissect and cut off the mesoappendix and appendicular artery using the IT knife or hook knife; 4) snareassisted traction of the partially dissected appendix for continuous dissection; 5) closure of the defect using double endoscopic suture technique after repeated cleansing of the resected area. A nasogastric tube was inserted into the rectum for anal decompression and removed after 2-3 days. After the procedure, patients remained fasted for at least 3 days and received intravenous antibiotics during this period.

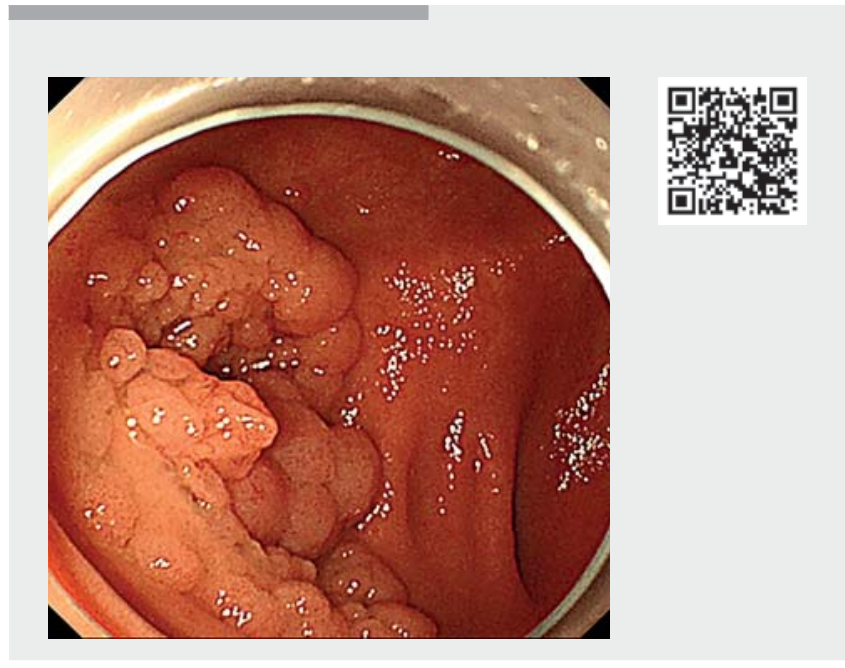

Video 1 The endoscopic transcecal appendectomy procedure.

Online content viewable at:

https://doi.org/10.1055/a-1675-2625

\section{Outcomes and definitions}

The primary outcome of the study was technical success of ETA. Secondary outcomes included postoperative adverse events, postoperative hospital stay, and recurrence. Technical success was defined as successful en bloc resection and $R 0$ resection of neoplastic lesions, and successful en bloc resection of non-neoplastic lesions. En bloc resection was defined as resection of the lesion and the appendix. R0 resection was defined as complete resection with negative margins. Postoperative adverse events included postoperative bleeding, perforation, and intra-abdominal abscess (IAA). Postoperative bleeding was defined as hemorrhage with clinical symptoms and confirmed by emergency endoscopy from the time of procedure completion to postoperative day 28 [13]. Postoperative perforation was defined as perforation of the cecum. IAA was defined as abscess collection inside the abdominal cavity confirmed by abdominal ultrasound or computed tomography scan. Recurrence was defined as neoplastic lesions recurring in the same location [14]. All cases were followed until April 2021.

\section{Statistical analysis}

Statistical analyses were conducted using SPSS version 25.0 (IBM Corp., Armonk, New York, USA). Continuous variables were expressed as median and range. Categorical variables were expressed as counts and percentages.

\section{Results}

A total of 13 patients with lesions at the appendiceal orifice underwent ETA during the study period. The characteristics of patients, lesions, and outcomes are shown in $>$ Table 1 . There were six male and seven female patients. Ages ranged from 33 to 87 years, with a median age of 64 years. Six patients had comorbidities and three patients had previous history of abdomi- 

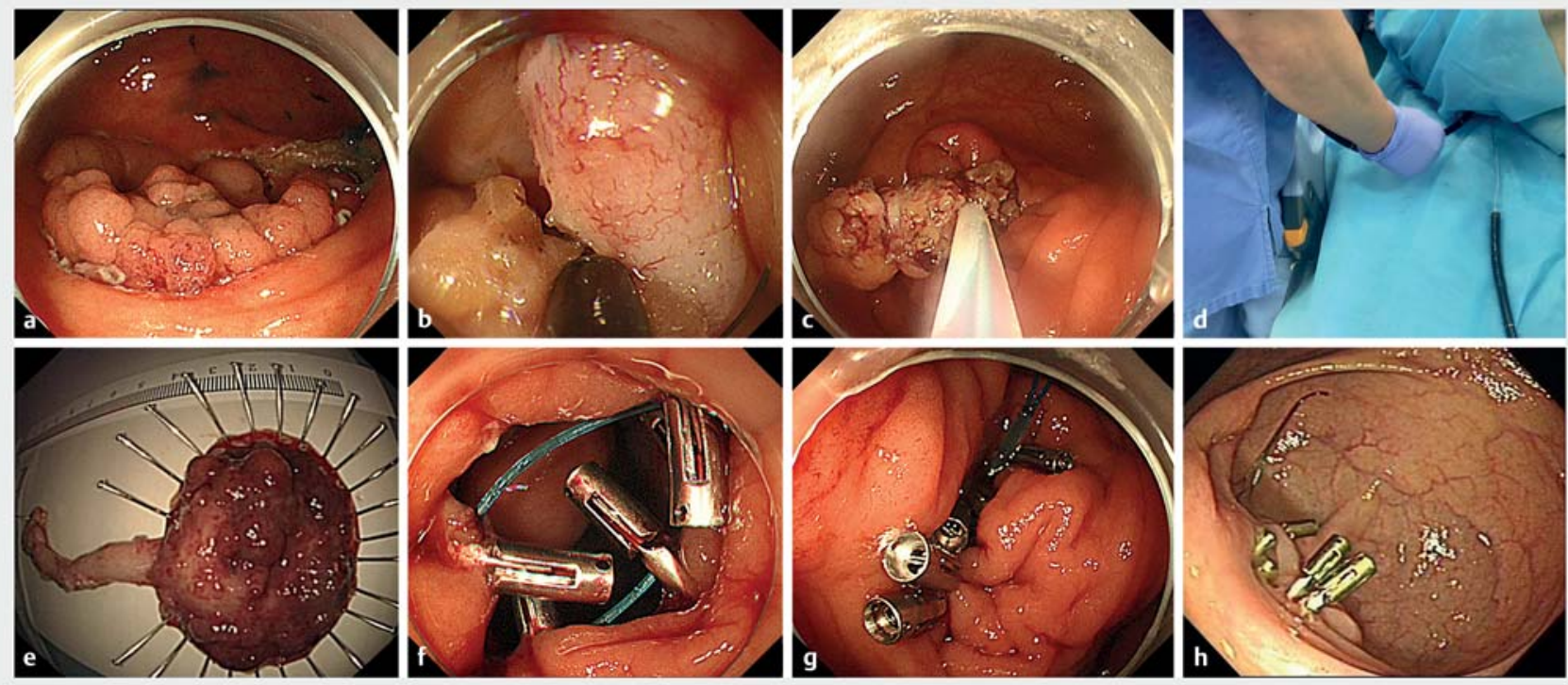

- Fig. 2 Endoscopic images of endoscopic transcecal appendectomy. a Near-circumferential full-thickness resection around the appendiceal lesion after marking. b Dissection of the mesoappendix and appendicular artery. c Snare-assisted traction of the partially dissected appendix for adequate exposure of the cutting line. $\mathbf{d}$ A second endoscope was inserted for continuous dissection. $\mathbf{e}$ The resected lesion and appendix. $\mathbf{f}$ Initial closure of the defect using purse-string suture technique. $\mathbf{g}$ Secondary closure of the defect using endoclips. $\mathbf{h}$ The healing of the defect after 1 month, with residual endoloop and endoclips.

nal surgery. Under colonoscopy, five lesions manifested as polypoid lesions, four lesions were LSTs, and four lesions were SMLs (see Fig. $1 \mathrm{~s}$ in the online-only Supplementary material). The median lesion size was $20 \mathrm{~mm}$ (range 8-50). Histopathological analysis showed four adenomas, two serrated lesions, two high grade intraepithelial neoplasias, one low grade intraepithelial neoplasia, one low grade appendiceal mucinous neoplasm, and three cases of appendicitis.

Technical success was achieved in all 13 patients. The median procedure time was 167 minutes (range 90-220). The median fasting time and postoperative hospital stay were 4 days (range 3-13) and 8 days (range 6-18), respectively. There were no cases of postoperative bleeding, perforation, or IAA. The median medical cost during the whole hospitalization was 37219 yuan (range 31 206-53 450). During a median followup of 17 months (range 1-28), no recurrence was detected.

\section{Discussion}

This retrospective case series study showed that all 13 appendiceal orifice lesions were successfully resected using the ETA technique, without postoperative bleeding, perforation, or IAA, demonstrating that ETA is a feasible, safe, and effective technique for the treatment of appendiceal orifice lesions.

With increased colon cancer screening, appendiceal and/or cecal lesions involving the appendiceal orifice are becoming more frequently encountered [15]. Surgery, including right hemicolectomy and partial cecectomy, has often been used as the standard therapy for these lesions. However, right hemicolectomy is associated with relatively high postoperative complications and may be considered excessive for relatively benign lesions such as adenomas, serrated lesions, and low grade appendiceal mucinous neoplasms $[5,16]$. Partial cecectomy can be less invasive than hemicolectomy, but it is difficult for the surgeon to visualize the lesion margins, thus extended resection or even right hemicolectomy may be performed in certain cases to ensure negative margins are obtained [5]. In addition, conversion from laparoscopic to open surgery may be needed in some cases, which further increases medical costs and surgical trauma [17]. Compared with surgery, ETA has several potential advantages. First, endoscopists can directly visualize the extent of the appendiceal orifice lesion, which could allow maximum preservation of the ileocecal valve and intestine. Second, endoscopists have more direct access to the appendiceal orifice lesion and the appendix, which could facilitate the identification of the appendix and reduce potential injuries to surrounding tissues, especially in patients with previous abdominal surgery. Third, the ETA technique leaves no scar on the abdomen and has no complications associated with surgical incision, such as incisional hernia and wound infection [18].

Endoscopic procedures such as EMR, ESD, and EFTR are also alternative treatments for appendiceal orifice lesions [2-4,68]. However, it is not appropriate to perform EMR if the lesion margin inside the appendiceal orifice cannot be visualized or if more than $50 \%$ of the circumference of the appendiceal orifice is involved [2]. R0 resection is often not achievable using the conventional ESD technique if the lesion extends deeply into the appendiceal orifice or if the lesion involves more than $75 \%$ of the appendiceal orifice circumference [3,4]. Traction-assisted ESD can achieve higher R0 resection rates (more than $80 \%$ ) for lesions extending into the appendiceal orifice, with short procedure times and length of stay, but additional surgery is re- 
- Table 1 Characteristics of the patients, lesions, and outcomes $(n=13)$.

\begin{tabular}{|c|c|}
\hline Age, median (range), years & $64(33-87)$ \\
\hline \multicolumn{2}{|l|}{ Sex, n (\%) } \\
\hline - Male & $6(46)$ \\
\hline - Female & $7(54)$ \\
\hline Comorbidity, n (\%) 1 & $6(46)$ \\
\hline Previous abdominal surgery, $\mathrm{n}(\%)^{2}$ & $3(23)$ \\
\hline \multicolumn{2}{|l|}{ Lesion type, n (\%) } \\
\hline - Polypoid lesion & $5(38)$ \\
\hline - Laterally spreading tumor & $4(31)$ \\
\hline - Submucosal lesion & $4(31)$ \\
\hline Lesion size, median (range), mm & $20(8-50)$ \\
\hline \multicolumn{2}{|l|}{ Histology, n (\%) } \\
\hline - Adenoma & $4(31)$ \\
\hline - Sessile serrated lesion & $2(15)$ \\
\hline - High grade intraepithelial neoplasia & $2(15)$ \\
\hline - Low grade intraepithelial neoplasia & $1(8)$ \\
\hline - Low grade appendiceal mucinous neoplasm (Tis) & $1(8)$ \\
\hline - Appendicitis with abscess or cyst & $3(23)$ \\
\hline Technical success, $\mathrm{n}(\%)$ & $13(100)$ \\
\hline Postoperative adverse events, n (\%) & $0(0)$ \\
\hline Procedure time, median (range), minutes & $167(90-220)$ \\
\hline Fasting time, median (range), days & $4(3-13)$ \\
\hline Postoperative hospital stays, median (range), days & $8(6-18)$ \\
\hline Medical cost, median (range), yuan & $\begin{array}{l}37219 \\
(31206- \\
53450)\end{array}$ \\
\hline Follow-up, median (range), months & $17(1-28)$ \\
\hline Recurrence, n (\%) & $0(0)$ \\
\hline \multicolumn{2}{|c|}{$\begin{array}{l}1 \text { Comorbidity including hypertension, coronary heart disease, asthma, hy- } \\
\text { pothyroidism, and diabetes. } \\
2 \text { Previous surgery including surgery for rectal cancer and sigmoid colon } \\
\text { cancer. }\end{array}$} \\
\hline
\end{tabular}

quired in some of these patients, especially for patients with deep invasion without previous appendectomy $(27.3 \%, 3 / 11)$ [19]. Recently reported single-step, non-exposure EFTR allows only partial resection of the appendix [6-8], and thus residual lesion tissue may remain. Reported rates of $\mathrm{R} 0$ resection using EFTR ranged from $64 \%$ to $93 \%$ [6-8]. In addition, postoperative appendicitis may develop when using ESD or ETFR for appendiceal orifice lesions [3,6-8]. In contrast, ETA can achieve complete resection of the lesion and the appendix simultaneously, avoiding residual lesion tissue and postoperative appendicitis. In the present study, complete resection was achieved in all cases without postoperative adverse events, providing direct evidence of the feasibility, safety, and effectiveness of the ETA technique for appendiceal orifice lesions.

There are several points to note when performing the ETA procedure. First, prudent management of the appendiceal artery helps to prevent intraprocedural bleeding. The location of the appendiceal artery in the mesoappendix is variable. Precise mesoappendix resection is important for prevention of accidental injury to the appendiceal artery, especially when the mesoappendix is relatively thick. Sufficient exposure of the appendiceal artery before coagulation is recommended in order to achieve desirable hemostasis. Second, it is difficult to resect fat tissue in the mesoappendix, which may be due to higher electrical resistance in fat [20]. Third, endoscopic intervention can be intrinsically challenging in this location as any distal looping hinders endoscope maneuverability.

It is worth noting that tumor seeding may develop during ETA. The neoplasm should be kept intact during the whole procedure. Near-circumferential full-thickness resection around the lesion may help to reduce the risk of tumor seeding. Application of the snare to pull the lesion into the gut may further help to minimize the possibility of tumor seeding. For now, we recommend that the ETA technique be performed with great caution in patients with precancerous lesions or low grade malignant neoplasms that involve the appendiceal orifice, and it should not be performed for appendiceal orifice lesions with deep infiltration. Therefore, detailed preoperative evaluations such as endoscopy, endoscopic ultrasound, and computed tomography are needed to exclude lesions with high malignancy. In addition to preoperative assessment, detailed evaluation of the resected lesion and appendix should be performed to guide postoperative management for these patients undergoing ETA. Close follow-up should also be performed to assess the longterm outcomes of the ETA technique. In this study, there was no tumor recurrence during a median follow-up of 17 months, providing preliminary evidence of the oncological safety of ETA for appendiceal orifice lesions; however, long-term follow-up results are needed to further confirm our findings.

There are several limitations to the study. First, the study was a retrospective case series study. Although we consecutively collected all cases undergoing ETA in our hospital, and the cases comprised patients with different demographic features, and lesion morphology and pathological types, the risk of selective bias could not be totally ruled out. Second, the sample size was relatively small for full evaluation of this novel endoscopic approach. Further prospective studies with larger sample sizes are needed to further assess the safety and effectiveness of the ETA procedure for appendiceal orifice lesions. Third, we did not present intraprocedural bleeding findings. This is mainly because intraprocedural bleeding occurred in all ETA procedures; however, intraprocedural bleeding was less than $20 \mathrm{~mL}$ in all cases and was easily controlled by endoscopic coagulation. Finally, all ETA procedures were performed by a single advanced endoscopist, and the findings cannot therefore be generalized to other or less experienced endoscopists.

In conclusion, ETA was shown to be feasible, safe, and effective in the management of appendiceal orifice lesions. Large, 
multicenter, prospective studies are needed to further assess this technique.

\section{Acknowledgment}

This research was funded by National Natural Science Foundation of China (Grant No: 82170675 ) and 1.3 .5 project for disciplines of excellence, West China Hospital, Sichuan University (Grant No: ZYJC21011).

Funding

1.3.5 project for disciplines of excellence, West China Hospital, Sichuan University ZYJC21011

National Natural Science Foundation of China 82170675

Competing interests

The authors declare that they have no conflict of interest.

\section{References}

[1] Tanaka S, Kashida H, Saito Y et al. JGES guidelines for colorectal endoscopic submucosal dissection/endoscopic mucosal resection. Dig Endosc 2015; 27: 417-434

[2] Tate DJ, Desomer L, Awadie $\mathrm{H}$ et al. EMR of laterally spreading lesions around or involving the appendiceal orifice: technique, risk factors for failure, and outcomes of a tertiary referral cohort (with video). Gastrointest Endosc 2018; 87: 1279-1288

[3] Jacob H, Toyonaga T, Ohara Y et al. Endoscopic submucosal dissection of cecal lesions in proximity to the appendiceal orifice. Endoscopy 2016; 48: 829-836

[4] Song EM, Yang HJ, Lee HJ et al. Endoscopic resection of cecal polyps involving the appendiceal orifice: a KASID multicenter study. Dig Dis Sci 2017; 62: 3138-3148

[5] Kulaylat AS, Boltz MM, Moyer M et al. Management of large cecal polyps: when can the ileocecal valve be spared? Dis Colon Rectum 2018; 61: 1089-1095
[6] Bronzwaer MES, Bastiaansen BA], Koens L et al. Endoscopic full-thickness resection of polyps involving the appendiceal orifice: a prospective observational case study. Endosc Int Open 2018; 6: E1112-E1119

[7] Ichkhanian Y, Barawi M, Seoud T et al. Endoscopic full-thickness resection of polyps involving the appendiceal orifice: a multicenter international experience. Endoscopy 2021: doi:10.1055/a-1345-0044

[8] Schmidbaur S, Wannhoff A, Walter B et al. Risk of appendicitis after endoscopic full-thickness resection of lesions involving the appendiceal orifice: a retrospective analysis. Endoscopy 2021; 53: 424-428

[9] Liu BR, Song JT, Liu ZH et al. Endoscopic transcecal appendectomy: the first human case report. Gastrointest Endosc 2018; 87: 311-312

[10] Chen T, Xu A, Lian J et al. Transcolonic endoscopic appendectomy: a novel natural orifice transluminal endoscopic surgery (NOTES) technique for the sessile serrated lesions involving the appendiceal orifice. Gut 2021; 70: 1812-1814

[11] Yuan XL, Cheung O, Du J et al. Endoscopic transcecal appendectomy. Endoscopy 2019; 51: 994-995

[12] Liu BR, Ullah S, Ye L et al. Endoscopic transcecal appendectomy: a novel option for the treatment of appendiceal polyps. VideoGIE 2019; 4: 271-273

[13] Mochizuki S, Uedo N, Oda I et al. Scheduled second-look endoscopy is not recommended after endoscopic submucosal dissection for gastric neoplasms (the SAFE trial): a multicentre prospective randomised controlled non-inferiority trial. Gut 2015; 64: 397-405

[14] Cotton PB, Eisen GM, Aabakken L et al. A lexicon for endoscopic adverse events: report of an ASGE workshop. Gastrointest Endosc 2010; 71: 446-454

[15] Tang S], Wu R. Ilececum: a comprehensive review. Can J Gastroenterol Hepatol 2019; 2019: 1451835

[16] Young S, Sueda SK, Hotta M et al. Surgical management of appendiceal mucinous neoplasm: is appendectomy sufficient? J Surg Oncol 2020: $122: 1173-1178$

[17] Sakpal SV, Bindra SS, Chamberlain RS. Laparoscopic appendectomy conversion rates two decades later: an analysis of surgeon and patient-specific factors resulting in open conversion. J Surg Res 2012; 176: $42-49$

[18] Kim HS, Kim HG, Yang SY et al. Single-incision laparoscopic surgery compared to conventional laparoscopic surgery for appendiceal mucocele: a series of 116 patients. Surg Endosc 2021: doi:10.1007/ s00464-020-08263-3

[19] Oung B, Rivory J, Chabrun E et al. ESD with double clips and rubber band traction of neoplastic lesions developed in the appendiceal orifice is effective and safe. Endosc Int Open 2020; 8: E388-E395

[20] Curtiss LE. High frequency currents in endoscopy: a review of principles and precautions. Gastrointest Endosc 1973; 20: 9-12 